\title{
MicroRNA-202 induces myoblast to myocyte differentiation through targeting Rock-1
}

\author{
Maryam Honardoost ${ }^{1 *}$, Mahsa Bourbour ${ }^{1}$, Ehsan Arefian $^{2}$ \\ ${ }^{1}$ Maryam Honardoost, Endocrine Research Center, Institute of Endocrinology and Metabolism, Iran University of \\ Medical Sciences, Tehran, Iran, honardoost.m@iums.ac.ir \\ ${ }^{1}$ Mahsa Bourbour, Endocrine Research Center, Institute of Endocrinology and Metabolism, Iran University of \\ Medical Sciences, Tehran, Iran, afrang.negin@strc.ac.ir \\ ${ }^{2}$ Ehsan Arefian, Department of Microbiology, School of Biology, College of Science, University of Tehran, Tehran, \\ Iran, arfefian@ut.ac.ir
}

\section{*Corresponding Author:}

Maryam Honardoost, Assistant professor of Molecular Medicine, Endocrine Research Center, Institute of Endocrinology and Metabolism, Iran University of Medical Sciences, Tehran,

Address; No 10, Firoozeh St, Vali-asr Sq,Tehran, Iran.

Website; http://www.iums.ac.ir/link/iem

Tel: +982188945246

Email: $\underline{\text { honardoost.m@iums.ac.ir }}$ maryamil79@gmail.com 


\title{
MicroRNA-202 induces myoblast to myocyte differentiation through targeting Rock-1
}

\begin{abstract}
The expression patterns of microRNAs (small non-coding RNAs) are altered in many biological processes such as myogenesis. In this study, we aimed to investigate the impact of predicted miR-202, its target genes Akt2 and Rock-1 as a potential regulators of myoblast in myocyte differentiation process using $\mathrm{C} 2 \mathrm{C} 12$ cell line. After confirmation of the differentiation process induced by $3 \%$ horse serum, the expression level of miRNA and its targets were evaluated. In the following, luciferase assay was conducted to approve the effect of miRNA on its target. Our results indicated that miR-202 and Akt2 were significantly up-regulated during differentiation, while Rock-1 was downregulated. Co-transfection of miRNA with psiCHECK2-Rock-1 significantly presented that Rock-1 was directly targeted by miR-202. On the contrary, miR-202 has failed to enforce its inhibitory effect on Akt2 expression. In particular, miR-202 seems to be a regulator of muscle differentiation pathway thought targeting Rock-1.
\end{abstract}

Keywords: miR-202, Rock-1, C2C12, Muscle, Differentiation, 


\section{Introduction}

Myogenesis is a specific multi-step differentiation process that is performed in embryonic and postnatal stages. This process is regulated by a multitude of external and internal markers which activated particular proteins including MyoD and Myf5 and controlled by a diverse group of signaling pathways such as PI3K/AKT and Rho family G proteins pathways [1-3].

The PI3K/AKT signaling pathway is an essential couple of differentiation process which is regulated by IGFs and controls muscle development. Previous data suggest that AKT2 as a down steam component of PI3K cascade plays a vital role in myogenic differentiation [4]. AKT2 motivates MyoD transactivation and consequently increases myogenin transcription [5, 6] or inhibits apoptosis during the differentiation process [5-8].

The Rho family $G$ proteins pathway is the other functional pathway in myogenesis $[8,9]$. It has been reported that Rho-associated kinase 1(ROCK-1), as a downstream effector of Rho GTPases, has a negative regulatory effect on muscle development [10]. Recently, it has been shown that ROCK inhibition allows myoblast fusion, therefore, accelerates myogenesis $[8,10,11]$.

Complicated controlling mechanisms of mentioned signaling pathways are applied by various factors during muscle development. Seeking to discover the regulatory effects of noncoding RNAs, investigators are interested in discovering microRNAs and their function in myogenesis [12].

MicroRNAs are small, conserved noncoding RNAs with 18-22 nucleotides [13] which are known as a new class of gene regulatory factors. They bind to the 3'untranslated region (UTR) of targeted mRNA $[14,15]$. As a result of complementary binding, translation of their relevant protein is inhibited. Accordingly, multiple molecular and cellular mechanisms have been reported to be under influence of miRNAs and their aberrant expression could be a major reason for cellular dysfunctions [16, 17]. Lately, researchers have determined some of the particular miRNAs as an inhibitory factor of differentiation and some others as an activator. However, several studies are required to find out about the function of miRNAs in myogenesis. This study aims to investigate the impact of microRNA-202 as a regulator of Akt2 and ROCK in myogenesis. 


\section{Materials and Methods}

\subsection{Bioinformatics analysis}

We used three major algorithms, Target Scan 6.2 [18], miRWalk [19], and RNAhybrid [20] to determine the specific targets of miR-202. Selected targets pick out from those sample targets which had the top score in at least two prediction lists.

\subsection{Cell culture}

C2C12 cells were grown in growth medium (GM; Dulbecco's Modified Eagle Medium and 10\% fetal bovine serum (Gibco, UK)) and when they reach 90\% confluency, GM medium was replaced with differentiation medium (DM; Dulbecco's Modified Eagle Medium containing 3\% horse serum (Gibco, UK)). The differentiation procedure was continued for 72 hours. Similarly, our control cells were cultured just in GM for 72 hours. It is notable to mention that all types of cell cultures were repeated at least three times.

\subsection{Immunocytochemistry (ICC)}

After the C2C12 differentiation (72 hours), Immunocytochemical detection of specific markers was performed by using anti-Pax7 or anti-myosin antibody (Sigma, USA) as previously described (data not shown).

\subsection{RNA isolation and quantitative real-time PCR}

RNA extraction was performed using TRIzol reagent (Invitrogen, USA) and cDNA was synthesized according to reverse transcription kit (Fermentas, USA) using random hexamer for genes and stem-loop primer for miRNAs. Quantitative real-time polymerase chain reaction (PCR) was applied as previously described [21, 22]. B-actin and Snord 47(U47) were selected as the internal control for the targeted gene and miRNA, respectively. The method to clarify gene expression level was the $2^{-\Delta \Delta C t}$.

\subsection{Comparison of real-time PCR result by a high-throughput method data}

GEO, accession \#GSE4694 row data was download from NCBI databank [23] and analyzed to compare with our real-time PCR results. 


\subsection{Luciferase assay}

The 3'UTR sequence of Rock-1 was cloned into psiCHECK-2 luciferase reporter plasmid. After seeding $20 \times 10^{4} 293$ T cells in 96-well plates, psi-CHECK-Rock-1 and PCDH-miR-202 were cotransfected using lipofectamine 3000 methods, and Luciferase activity was estimated after 48 hours using Dual-Luciferase Reporter Assay System (Promega). Renilla luciferase signal was standardized to the Firefly luciferase signal activity to control transfection effectiveness.

\subsection{Statistical analysis}

The data are presented as mean \pm standard error. REST analysis and Student's t-test were applied to determine statistical significance and the criterion for significance was set at $\mathrm{P}<0.05$.

\section{Results}

\subsection{Bioinformatics analysis}

According to the results of bioinformatics prediction, we selected miR-202 and its targets, Akt2 and Rock-1 for this study, which in our prediction methods had the best miRNA-mRNA interaction (Table 1). 
Table 1. Characteristics of miR-202 and its predicted targets

\begin{tabular}{lll}
\hline Gene Name & Predicted base pair site & Position site/Number of paired bases \\
\hline Rock-1 & 5'- CAUAGGAA-3' & $1213-1220 / 8 \mathrm{mer}$ \\
hsa-miR-202 & 3'- GUAUCCUU-5' & \\
& & \\
Akt2 & 5'- UACCUCA-3' & $2617-2623 / 7 \mathrm{mer}$ \\
hsa-miR-202 & 3'- AUGGAG-5' & \\
\hline
\end{tabular}

\subsection{Gene expression level}

Quantitative real-time PCR (q-RT PCR) was applied to evaluate miR-202, Akt, and Rock-1 expression levels in differentiated (myocyte) vs undifferentiated (myoblast) cell lines. As miR202 increased in myocytes (Figure 1a), Roke-1 was down-regulated, while Akt2 expression level was ascended (Figure 1b). Although bioinformatics described Akt2 as the target of miR202 , its increase could be due to other molecular pathways that have helped differentiate muscles $[24,25]$.
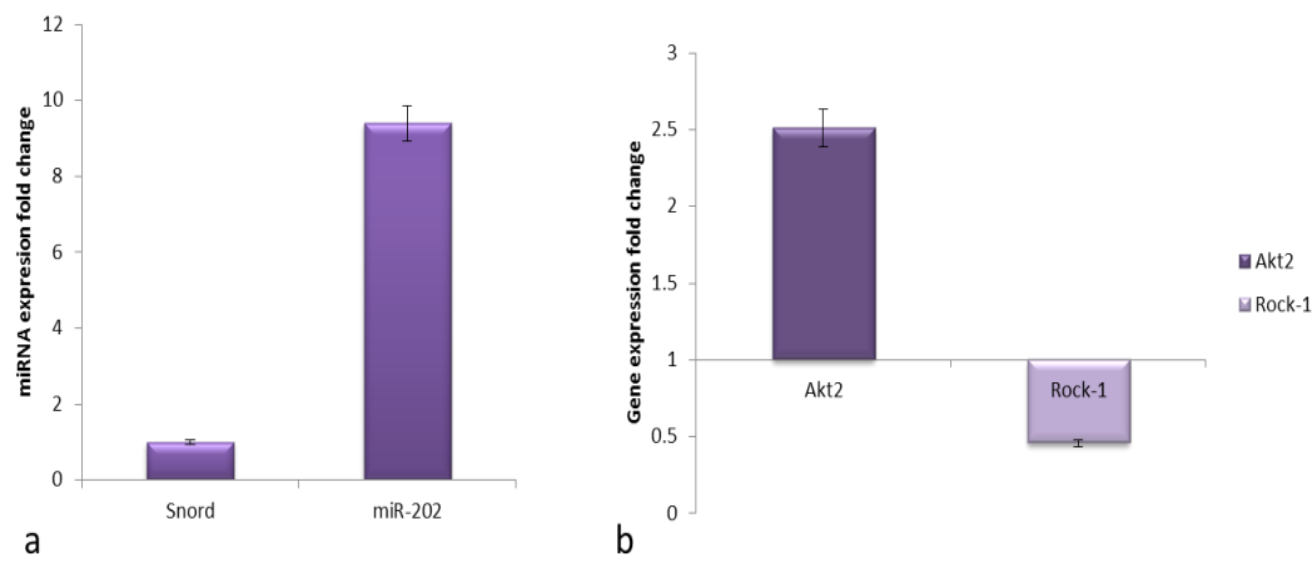

Figure 1: Expression pattern of miR-202 (1a) and its target genes during myoblast differentiation (1b). Based on qRT-PCR results, while Rock-1 was down-regulated (1b), Akt2 was up-regulated during the differentiation process $(\mathrm{P}-\mathrm{value} \leq 0.05)$. Error bars indicate $\mathrm{SEM}(\mathrm{n}=3)(\mathrm{P}<0.05)$. 


\subsection{Comparison of real-time PCR result by a high-throughput method data}

Validation of our q-RT PCR results was achieved by micro-array analysis results. Both analyses were tracing the same way in gene`s up/down-regulation (Figure 2).

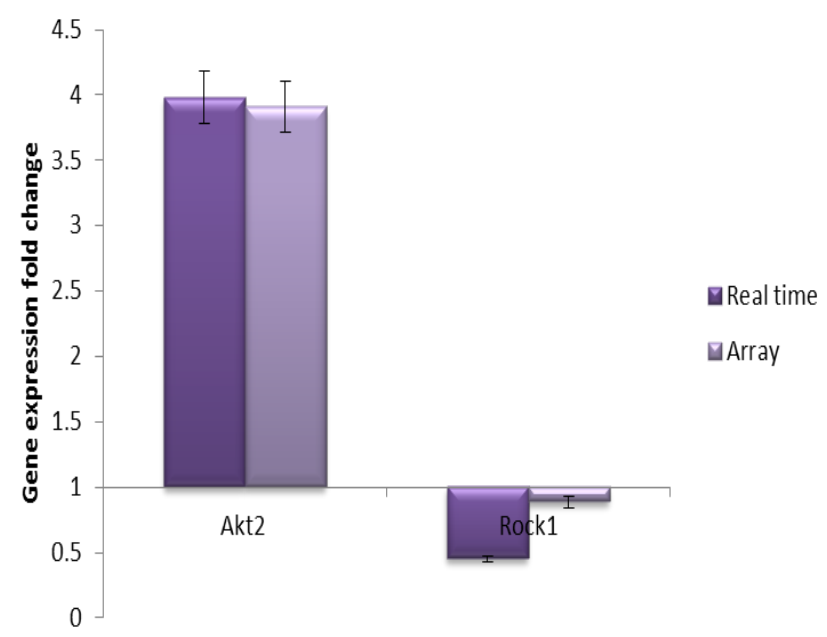

Figure 2. Comparison of the expression levels of predicted targets during myogenesis based on qRT-PCR and microarray analysis. The data were consistent between the two methods.

\subsection{Luciferase assay}

Based on our bioinformatics results, we hypothesized that miR-202 may have a positive effect on muscle differentiation through ROCK-1 down-regulation. For evaluating the hypothesis, we constructed a Luciferase reporter vector that carries Rock-1 3 `UTRs downstream of luciferase stop codon psiCheck2. The results have been shown that co-transfection of miR-202 with psiCheck2-ROCK-1, significantly down-regulated luciferase expression (approximately 40\%). The result confirmed that ROCK-1 was directly targeted by miR-202 (Figure 3). 


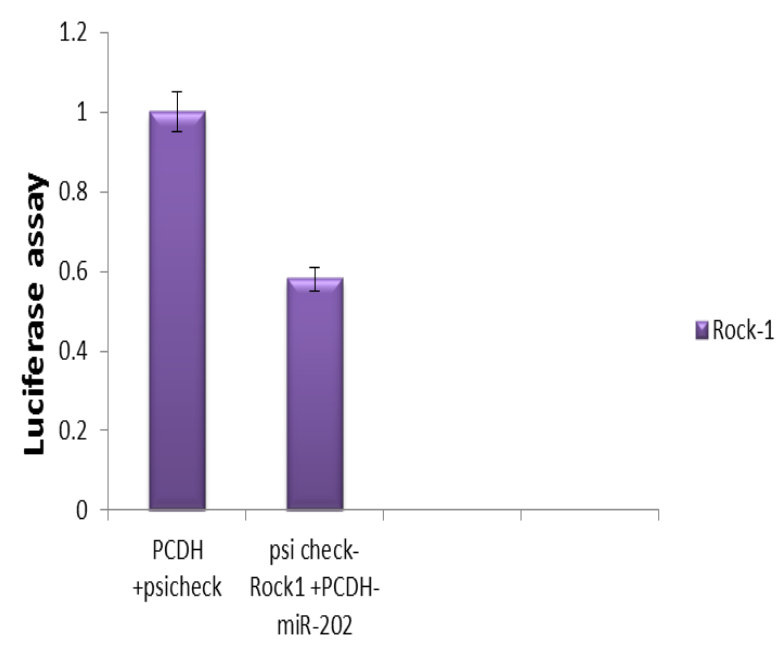

Figure 3. miR-202 has complementary sequences in Rock-1 -3`UTRs and targets it. Co-transfection of miRNA with psiCheck2-target significantly down-regulated luciferase expression. Error bars indicate $\operatorname{SEM}(n=3) .(P<0.05)$.

\section{Discussion}

MicroRNAs are small biomolecules regulating different cellular and molecular pathways including muscle cell differentiation [26]. Several studies claim that miRNAs have a vital role in muscle differentiation [1]. Thus we have selected miR-202 as a novel predicted regulator of PI3K/AKT and Rho G proteins signaling components, Ak2 and ROCK-1, and evaluated the expression level of these molecules before and after myoblast to myocyte differentiation.

According to our survey, miR-202 had an increased level during muscle development. Interestingly, ROCK-1 has shown a reduced level, while Akt2 had an opposite expression pattern. To confirm the real-time results, using an Affymetrix cDNA microarray dataset of GEO accession \#GSE4694, we compared the fold change of $A k t 2$ and ROCK-1 in undifferentiated and differentiated C2C12 line. Data analysis revealed that the expression pattern of ROCK-1 and Akt 2 were the same in both methods (P-value $\leq 0.05$ ), however, the fold change of ROCK-1 was shown to be more decreased in microarray analysis comparison with qRT-PCR analysis (Figure 2).

Furthermore, the result of our luciferase assay confirmed that ROCK-1 is directly inhibited through miR-202 induction (Figure 3). Recently, it has been shown that preventing ROCK-1 by its inhibitor, increased the phosphorylation level of IRS1/2 tyrosine and consequently activated myogenesis through PI3K activation, a major differentiation pathway in myogenesis [27, 28]. 
Other studies proved that ROCK-1 is the final inhibitor for differentiating myoblasts to myocyte which report distinguished the association between ROCK-1 and mTOR signaling pathway in suppressing the muscle-cell differentiating process $[29,30]$.

Moreover, it has been claimed that evaluating miRNAs could be a useful marker in various steps of the myocyte differentiation pathway [22]. As a result of this, some researchers have reported the utilization of micro RNAs as an inducer of transforming myoblasts to myocytes such as miR148a. Zhang et al stated that miR-148a is up-regulated during the differentiation process. They have explained this overexpression due to inhibiting ROCK-1 in protein levels [29]. Hence, based on our results, it seems that miR-202 may be an effective regulator of myogenesis through direct suppression of ROCK- 1 and following indirect activation of PI3K signaling (Figure 4).

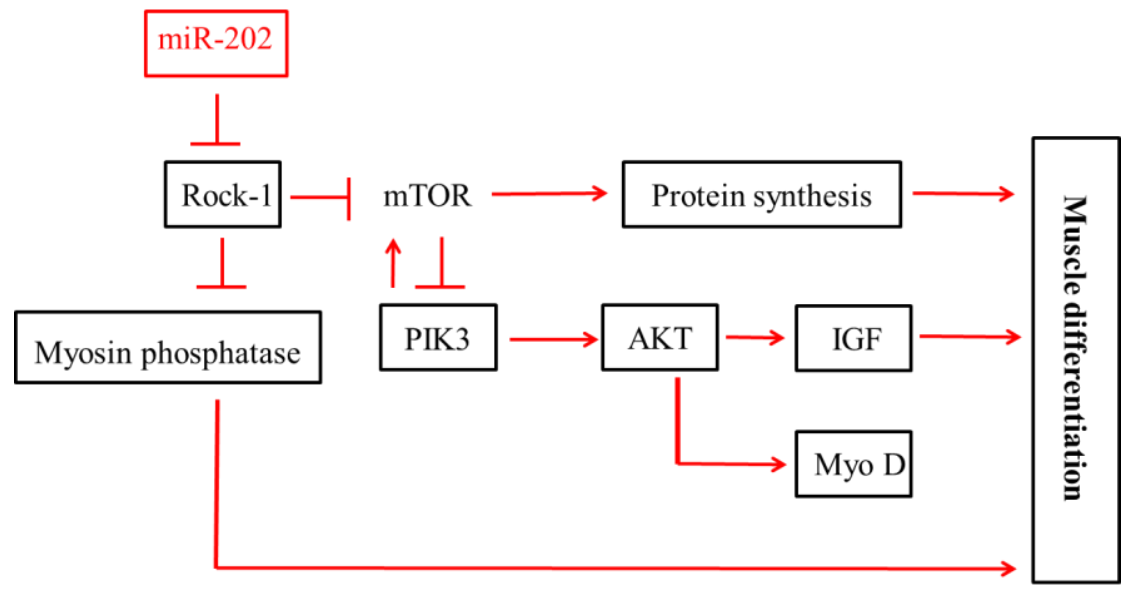

Figure 4. miR-202/ Rock-1/ PI3K relationship in the muscle differentiation process

However, our data has revealed that the other target of miR-202, Akt2, is deregulated during the differentiation process and it seems that miR-202 has failed to enforce its inhibitory effect on Akt2 expression. Although our bioinformatics prediction showed targeting of Akt2 mRNA by miR-202, the unexpected result is also justifiable.

As mentioned before, several studies have confirmed the positive role of PI3K signaling in myoblast differentiation, and the downstream genes of this pathway such as Akt2 were surveyed in various studies to differentiate their functions [31-33]. Recent studies have defined the specific expression of Akt2 in insulin-responsive tissues specifically skeletal muscle which creates the role of Akt2 in the muscle-cell differentiation process [34]. Furthermore, other published data has firmed the increased level of Akt2 in 10T1/2-MyoD cells, C2C12 myoblast, and Sol8 
myoblast, respectively [35-37]. Additionally, Sumitani et al reported Akt2 function due to its serine/threonine kinase activity stimulating myogenin $[6,38]$. It is also notable that Akt2 could induce IGF-II which is an activator for differentiating myoblasts to myotubes [39]. Moreover, Rotwein et al results have confirmed that inhibition of Akt2 in myoblasts causes myotube cells with the incomplete size of nuclei and the content of them [40]. Furthermore, Vandromme et al have suppressed the myogenesis process using Akt2 antibody [41].

Therefore, Akt-2 is a fundamental part of myogenesis and the cellular configuration is such that the expression of this gene increases [42]. Subsequently, do not mind if our examined microRNA could not inhibit Akt2 expression, while in other processes or cell types, it may be down-regulated by mentioned miRNA.

Since elucidating the molecular settings of cellular signaling helps to better understanding myogenesis, non-coding RNA such as miRNA could be a useful marker to identify the controlling mechanisms of muscle differentiation [43-45].

Our results may determine that Rock-1 inhibition through miR-202 could be one of the capable procedures involving in myogenesis. Accordingly, down-regulation of miR-202 expression may be considered as an aspect of producing induced pluripotent stem cells (iPSCs) from myocytes which have therapeutic advantages.

\section{Conclusion}

In this study, we demonstrated that miR-202 is up-regulated during the differentiation process which is responsible for ROCK-1 down-regulation. However, there should be multiple examinations due to confirmation of miR-202 function. Likewise, this study along with other researches could introduce the function and application of miRNAs in muscle development. Incorporation of several aspects via miRNAs' role in signaling pathways and possible treatments should be future study approaches.

\section{Conflict of interest}

The authors state that they have no conflict of interest.

\section{Acknowledgments}


This research was partially supported by the stem cell technology research center, Tehran, Iran. We thank Dr.Masoud Soleimani who provided insight and expertise that greatly assisted the research.

\section{References}

1. Bentzinger, C.F., Y.X. Wang, and M.A. Rudnicki, Building muscle: molecular regulation of myogenesis. Cold Spring Harb Perspect Biol, 2012. 4(2).

2. Yokoyama, S. and H. Asahara, The myogenic transcriptional network. Cell Mol Life Sci, 2011. 68(11): p. 1843-9.

3. Bentzinger, C.F., J. von Maltzahn, and M.A. Rudnicki, Extrinsic regulation of satellite cell specification. Stem Cell Res Ther, 2010. 1(3): p. 27.

4. Zhang, S. and N. Chen, Regulatory Role of MicroRNAs in Muscle Atrophy during Exercise Intervention. Int J Mol Sci, 2018. 19(2).

5. Kaneko, S., et al., Positive feedback regulation between Akt2 and MyoD during muscle differentiation. CLONING OF Akt2 PROMOTER (vol 277, pg 23230, 2002). Journal of Biological Chemistry, 2016. 291(43): p. 22850-22850.

6. Sumitani, S., et al., Akt1 and Akt2 differently regulate muscle creatine kinase and myogenin gene transcription in insulin-induced differentiation of C2C12 myoblasts. Endocrinology, 2002. 143(3): p. 820-8.

7. Jang, Y.N. and E.J. Baik, JAK-STAT pathway and myogenic differentiation. JAKSTAT, 2013. 2(2): p. e23282.

8. Komati, H., et al., Phospholipase $D$ is involved in myogenic differentiation through remodeling of actin cytoskeleton. Molecular Biology of the Cell, 2005. 16(3): p. 1232-1244.

9. Neri, L.M., et al., The nuclear phosphoinositide 3-kinase/AKT pathway: a new second messenger system. Biochim Biophys Acta, 2002. 1584(2-3): p. 73-80.

10. Castellani, L., et al., Fine regulation of RhoA and rock is required for skeletal muscle differentiation. Journal of Biological Chemistry, 2006. 281(22): p. 15249-15257.

11. Fortier, M., et al., RhoE controls myoblast alignment prior fusion through RhoA and ROCK. Cell Death and Differentiation, 2008. 15(8): p. 1221-1231.

12. Wang, X.H., MicroRNA in myogenesis and muscle atrophy. Curr Opin Clin Nutr Metab Care, 2013. 16(3): p. 258-66.

13. Liu, N., et al., The microRNA miR-34 modulates ageing and neurodegeneration in Drosophila. Nature, 2012. 482(7386): p. 519-U240.

14. Sweetman, D., et al., Specific requirements of MRFs for the expression of muscle specific microRNAs, miR-1, miR-206 and miR-133. Dev Biol, 2008. 321(2): p. 491-9.

15. Small, E.M., et al., Regulation of PI3-kinase/Akt signaling by muscle-enriched microRNA-486. Proc Natl Acad Sci U S A, 2010. 107(9): p. 4218-23.

16. Ge, Y. and J. Chen, MicroRNAs in skeletal myogenesis. Cell Cycle, 2011. 10(3): p. 441-8.

17. Honardoost, M., et al., Insulin Resistance Associated Genes and miRNAs. Applied Biochemistry and Biotechnology, 2014. 174(1): p. 63-80.

18. Lewis, B.P., C.B. Burge, and D.P. Bartel, Conserved seed pairing, often flanked by adenosines, indicates that thousands of human genes are microRNA targets. Cell, 2005. 120(1): p. 15-20.

19. Dweep, H., N. Gretz, and C. Sticht, miRWalk database for miRNA-target interactions. Methods Mol Biol, 2014. 1182: p. 289-305.

20. Kruger, J. and M. Rehmsmeier, RNAhybrid: microRNA target prediction easy, fast and flexible. Nucleic Acids Res, 2006. 34(Web Server issue): p. W451-4. 
21. Honardoost, M., et al., Development of Insulin Resistance through Induction of miRNA-135 in C2C12 Cells. Cell J, 2016. 18(3): p. 353-61.

22. Honardoost, M., et al., Expression Change of miR-214 and miR-135 during Muscle Differentiation. Cell J, 2015. 17(3): p. 461-70.

23. Chen I-HB, H.M., Guan T, Bubeck A, Gerace L, Nuclear envelope transmembrane proteins (NETs) that are up-regulated during myogenesis. BMC cell biology, 2006.

24. Chen, X., et al., PIP5K1alpha promotes myogenic differentiation via AKT activation and calcium release. Stem Cell Res Ther, 2018. 9(1): p. 33.

25. Zak, R.B., et al., Impact of local heating and cooling on skeletal muscle transcriptional response related to myogenesis and proteolysis. European Journal of Applied Physiology, 2018. 118(1): p. 101-109.

26. Horak, M., J. Novak, and J. Bienertova-Vasku, Muscle-specific microRNAs in skeletal muscle development. Dev Biol, 2016. 410(1): p. 1-13.

27. Charrasse, S., et al., RhoA GTPase regulates M-cadherin activity and myoblast fusion. Mol Biol Cell, 2006. 17(2): p. 749-59.

28. Wei, L., et al., Rho kinases play an obligatory role in vertebrate embryonic organogenesis. Development, 2001. 128(15): p. 2953-62.

29. Zhang, J., et al., MicroRNA-148a promotes myogenic differentiation by targeting the ROCK1 gene. J Biol Chem, 2012. 287(25): p. 21093-101.

30. Sun, Q., et al., Transforming growth factor-beta-regulated miR-24 promotes skeletal muscle differentiation. Nucleic Acids Res, 2008. 36(8): p. 2690-9.

31. Wei, W., et al., miR-29 targets Akt3 to reduce proliferation and facilitate differentiation of myoblasts in skeletal muscle development. Cell Death Dis, 2013. 4: p. e668.

32. Briata, P., et al., PI3K/AKT signaling determines a dynamic switch between distinct KSRP functions favoring skeletal myogenesis. Cell Death and Differentiation, 2012. 19(3): p. 478-487.

33. Li, J., et al., [Expression of Myogenin and MCK genes regulated by PI3K/AKT pathway]. Yi Chuan, 2013. 35(5): p. 637-42.

34. Motohashi, N., et al., Regulation of IRS1/Akt insulin signaling by microRNA-128a during myogenesis. J Cell Sci, 2013. 126(Pt 12): p. 2678-91.

35. Calera, M.R. and P.F. Pilch, Induction of Akt-2 correlates with differentiation in Sol8 muscle cells. Biochem Biophys Res Commun, 1998. 251(3): p. 835-41.

36. Altomare, D.A., et al., Cloning, chromosomal localization and expression analysis of the mouse Akt2 oncogene. Oncogene, 1995. 11(6): p. 1055-60.

37. Altomare, D.A., et al., Akt2 mRNA is highly expressed in embryonic brown fat and the AKT2 kinase is activated by insulin. Oncogene, 1998. 16(18): p. 2407-2411.

38. Small, E.M., et al., Regulation of PI3-kinase/Akt signaling by muscle-enriched microRNA-486. Proceedings of the National Academy of Sciences of the United States of America, 2010. 107(9): p. 4218-4223.

39. Erbay, E., et al., IGF-II transcription in skeletal myogenesis is controlled by mTOR and nutrients. Journal of Cell Biology, 2003. 163(5): p. 931-936.

40. Rotwein, P. and E.M. Wilson, Distinct actions of Akt1 and Akt2 in skeletal muscle differentiation. J Cell Physiol, 2009. 219(2): p. 503-11.

41. Vandromme, M., et al., Protein kinase $B$ beta/Akt2 plays a specific role in muscle differentiation. J Biol Chem, 2001. 276(11): p. 8173-9.

42. Ge, Y. and J. Chen, Mammalian target of rapamycin (mTOR) signaling network in skeletal myogenesis. J Biol Chem, 2012. 287(52): p. 43928-35.

43. Eisenberg, I., M.S. Alexander, and L.M. Kunkel, miRNAS in normal and diseased skeletal muscle. J Cell Mol Med, 2009. 13(1): p. 2-11. 
44. Luo, W., Q. Nie, and X. Zhang, MicroRNAs involved in skeletal muscle differentiation. J Genet Genomics, 2013. 40(3): p. 107-16.

45. van Rooij, E., et al., A family of microRNAs encoded by myosin genes governs myosin expression and muscle performance. Dev Cell, 2009. 17(5): p. 662-73. 